\title{
ChemComm
}

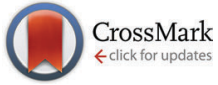

Cite this: Chem. Commun., 2015, 51, 9833

Received 8th April 2015,

Accepted 14th May 2015

DOI: $10.1039 / c 5 c c 02907 d$

www.rsc.org/chemcomm

\section{Lectin-gated, mesoporous, photofunctionalized glyconanoparticles for glutathione-responsive drug delivery $\dagger$}

\author{
Juan Zhou, ${ }^{a}$ Nanjing Hao, ${ }^{b}$ Thareendra De Zoyza, ${ }^{b}$ Mingdi Yan*ab and \\ Olof Ramström*a
}

\begin{abstract}
A stimuli-responsive drug delivery system based on fluorescent, lectin-gated, mesoporous glyconanoparticles has been developed and evaluated in normal- and cancer lung epithelial cells. The gating process proved efficient, exhibiting good sealing properties in the absence of the glutathione redox trigger, avoiding premature release in normal cells. In the presence of higher levels of glutathione in cancer cells, the lectin gate was rapidly opened and the anticancer drug released.
\end{abstract}

Nanomedical strategies with high therapeutic efficiencies and reduced adverse side-effects have attracted high interest in recent years. ${ }^{1}$ Controlled, stimuli-responsive drug release from nanocarriers, avoiding premature leakage, is in this context a key feature, ${ }^{2}$ and development of more efficient delivery systems based on nanomaterials is demanded. Mesoporous silica nanoparticles (MSNs) have demonstrated excellent potential as delivery containers, owing to their tunable pore sizes, high surface areas and overall biocompatibility. ${ }^{3}$ MSNs allow for straightforward, diffusion-controlled cargo entrapment and can be functionalized to release the loaded drug in response to chosen stimuli, such as light, ${ }^{4} \mathrm{pH},{ }^{5}$ temperature, ${ }^{6}$ enzyme action, ${ }^{7}$ and redox state. ${ }^{8}$ For the most part, these systems rely on non-proteinic structural elements, such as synthetic polymers and supramolecular assemblies, whereas the use of proteins as gatekeepers is still in early development. ${ }^{9}$ Proteins are however attractive as gating elements, since they are inherently biocompatible, can be of non-toxic nature, exist in many different shapes and sizes, and are amenable to a wide range of recognition systems. In the present study, we present an MSN-based, redox-controlled drug release system using protein-carbohydrate recognition as a gating factor.

\footnotetext{
${ }^{a}$ Department of Chemistry, KTH - Royal Institute of Technology, Teknikringen 30, S-10044 Stockholm, Sweden. E-mail: ramstrom@kth.se

${ }^{b}$ Department of Chemistry, University of Massachusetts Lowell, 1 University Avenue, Lowell, MA 01854, USA

$\dagger$ Electronic supplementary information (ESI) available: Experimental details and characterizations of functionalized particles, viability and confocal fluorescence images of cells incubated with FMSN. See DOI: 10.1039/c5cc02907d
}

Lectins are proteins of non-immune origin that interact with specific carbohydrate structures, thereby mediating ubiquitous important biological processes. ${ }^{10}$ One member of this family, the jack bean protein concanavalin A (Con A), is a homotetrameric protein at $\mathrm{pH}$ above 5.5 with a size of approximately $8 \mathrm{~nm} .{ }^{11}$ Con A binds primarily to $\alpha$-D-mannopyranosides with high specificities but relatively low affinities $\left(K_{\mathrm{a}}\right.$ for methyl $\alpha$-D-mannopyranoside is $8.2 \times 10^{3} \mathrm{M}^{-1}$ ) in the presence of $\mathrm{Ca}^{2+}$ and $\mathrm{Mn}^{2+}$ ions. ${ }^{12}$ The affinities can however be increased through multivalent interactions, ${ }^{13}$ often leading to considerably stronger binding than the corresponding monovalent carbohydrateprotein interactions. ${ }^{14}$ Since carbohydrate-based interactions are central in biological systems, glyconanomaterials have emerged as useful entities for biorecognition studies and different biomedical applications. ${ }^{15}$ These nanomaterials are functionalized with carbohydrate/glycan structures, thereby exerting interaction potential with biologically relevant blood-, cytosolicand cell-bound proteins.

Con A-gated MCM-41-type MSNs have been reported by Du and coworkers for controlled release of rhodamine $6 \mathrm{G}$ in vitro. ${ }^{9 c}$ Release was in this case effectuated by acidifying the solutions or by competitive binding with glucose. We however reasoned that redox control offer an efficient release mechanism, especially suited for targeting cancer cells. By engineering a disulfide linker into the gate function, the release would be responsive to the redox state of the cells, maintained by the glutathione/glutathione disulfide system. Healthy cells generally have considerably lower glutathione concentrations than cancer cells, thus enabling a selectivity effect between the different cells/tissues and low degrees of premature release. ${ }^{16}$

The gated nanomaterials were designed and fabricated as outlined in Scheme 1. Fluorescently-labeled mesoporous silica nanoparticles were chosen as carriers, further functionalized with D-mannose using a photochemical nitrene-mediated approach, and incorporating a redox-sensitive disulfide moiety in the linker. Doxorubicin, an anticancer drug with red fluorescence, was subsequently loaded into the nanopores, and the particles capped with Con A. Upon uptake by cancer cells, 

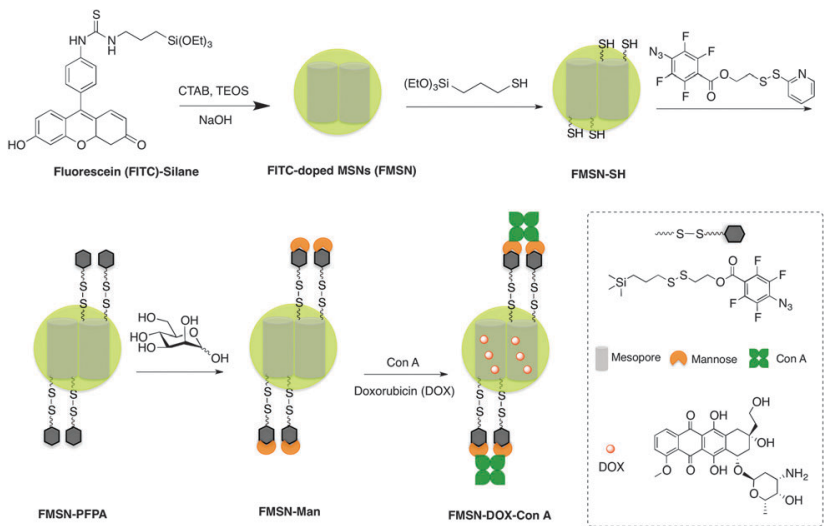

FMSN-PFPA

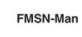

FMSN-DOX-Con A

Scheme 1 Synthesis of Con A-gated, FITC-doped, mesoporous silica glyconanoparticles.

doxorubicin would then be released owing to the glutathionemediated reduction of the disulfide bonds, thereby removing the Con A gatekeeper groups.

The fluorescein isothiocyanate- (FITC)-doped mesoporous silica nanoparticles (FMSN, Scheme 1) were prepared using a modified protocol of Lin and coworkers, involving a FITC-silane synthesized from fluorescein isothiocyanate and 3-aminopropyltriethoxysilane. ${ }^{17}$ The resulting MSNs displayed an average diameter of $130 \mathrm{~nm}$, as shown by transmission electron microscopy (TEM) (Fig. 1A and B), where the ordered lattice array indicated a uniform, well-defined mesoporous structure. Subsequent functionalization with 3-mercaptopropyltrimethoxysilane resulted in thiolated particles (FMSNSH, Scheme 1), as supported by a thiol absorption band around $2580 \mathrm{~cm}^{-1}$ in the Raman spectrum (Fig. S1, ESI $\dagger$ ).
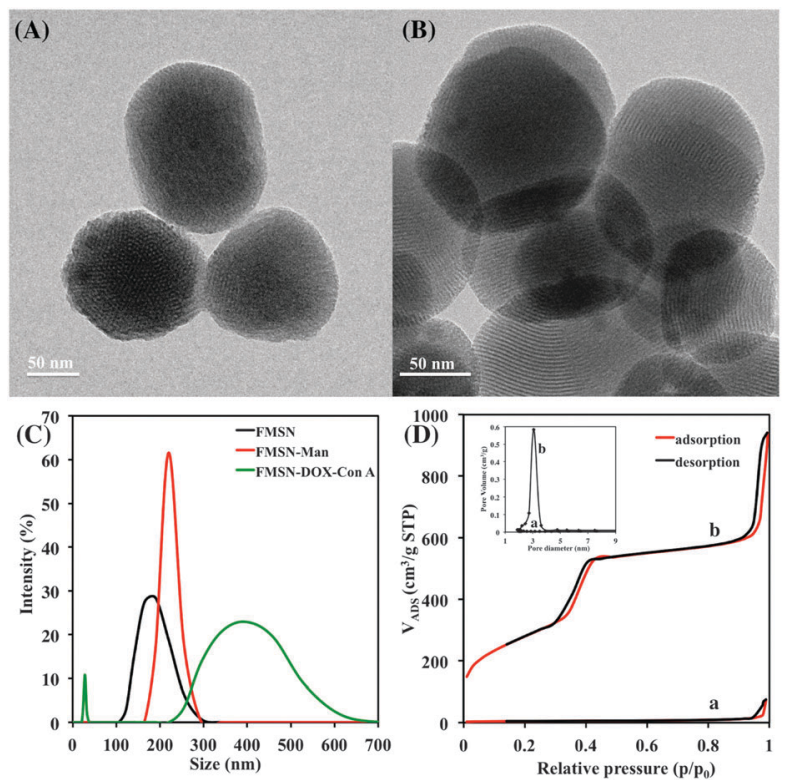

Fig. 1 TEM images of (A) FMSN- and (B) FMSN-DOX-ConA preparations: (C) size distribution of nanoparticles by DLS; (D) nitrogen adsorptiondesorption isotherms of (a) FMSN-DOX-ConA- and (b) FMSN samples; inset: corresponding pore size distribution of FMSN-DOX-Con A (a) and FMSN (b) particles.
Further modification using 2-(pyridin-2-yldisulfanyl)ethyl 4-azido-2,3,5,6-tetrafluorobenzoate yielded particles presenting disulfide-linked perfluorophenyl azide (PFPA) groups (FMSNPFPA, Scheme 1). Disulfide formation was supported by the azide group absorption at $2175 \mathrm{~cm}^{-1}$ in the FTIR spectrum, accompanied by the disappearance of the Raman thiol signal (Fig. S2, ESI $\dagger$ ). The particles were finally mannosylated under UV irradiation using a previously developed photocoupling method, ${ }^{18}$ where the PFPA azide moiety is converted into a nitrene that can undergo insertion into carbohydrate to give D-mannose-conjugated nanoparticles (FMSN-Man, Scheme 1). The carbohydrate content in FMSN-Man was estimated by an anthrone-based colorimetry assay, ${ }^{17 b, 19}$ indicating an approximate amount of $12 \mu \mathrm{g}$ of D-mannose per mg of particles (Fig. S3, ESI $\dagger$ ), and a coupling yield of $48 \%$.

The glyconanoparticles were next loaded with doxorubicin by gently stirring FMSN-Man suspensions in the presence of doxorubicin for $48 \mathrm{~h}$. The gatekeeper protein (Con A) was subsequently added, capping the D-mannose-functionalized nanoparticles through multivalent lectin-carbohydrate interactions, yielding the final drug delivery preparations (FMSN-DOX-ConA particles, Scheme 1). The amount of doxorubicin incorporated was estimated as $75 \mathrm{mg} \mathrm{g}^{-1}$ of particles, based on UV analysis before and after washing.

The particle synthesis, and the loading and gating process were also followed by dynamic light scattering (DLS, Fig. 1C). The FMSN particles displayed a hydrodynamic diameter of approximately $180 \mathrm{~nm}$, which increased slightly to $200 \mathrm{~nm}$ after PFPA functionalization and mannosylation. After doxorubicin loading and Con A capping, the size of the particles increased to $300-600 \mathrm{~nm}$, in part owing to particle crosslinking. The smaller particle population at around $30 \mathrm{~nm}$ is likely due to Con A aggregation under the experimental conditions used. The same pattern could be seen from the corresponding TEM images (Fig. 1B), where the capped FMSN-DOX-ConA particles displayed more aggregation than the original FITC-doped mesoporous silica nanoparticles.

As discussed in the above, the size of a Con A protomer is approximately $4 \mathrm{~nm}$ in one dimension, and the tetrameric protein close to twice that size. To confirm the gating potential of the lectin, Brunauer-Emmett-Teller (BET) surface area analysis and BarrettJoyner-Halenda (BJH) pore size and volume analysis were used to confirm the particles' mesoporous structure. As shown in Fig. 1D, the properties of the original FMSN preparation were typical to mesoporous silica particles with approximately $1020 \mathrm{~m}^{2} \mathrm{~g}^{-1}$ surface area and $3.1 \mathrm{~nm}$ pore diameter (b), while the Con A-capped FMSNDOX-ConA particles showed significantly decreased surface area and no obvious mesopores (a). This is owing to doxorubicin loading and lectin capping, similar to the results reported by Zhang and coworkers after $\beta$-cyclodextrin capping on DOX-loaded MSNs. ${ }^{20}$

The gating behaviour of the pores in the presence of glutathione was next evaluated. The mannosylated FMSN-Man particles were thus first capped with FITC-labeled Con A (yielding FMSN-fConA particles), and subsequently exposed to increasing concentrations of glutathione in PBS (pH 7.4). Upon incubation for $12 \mathrm{~h}$, the particles were removed by centrifugation, and the residual fluorescence emanating from reduction of the disulfide bond and release of the FITC-labeled Con A was 

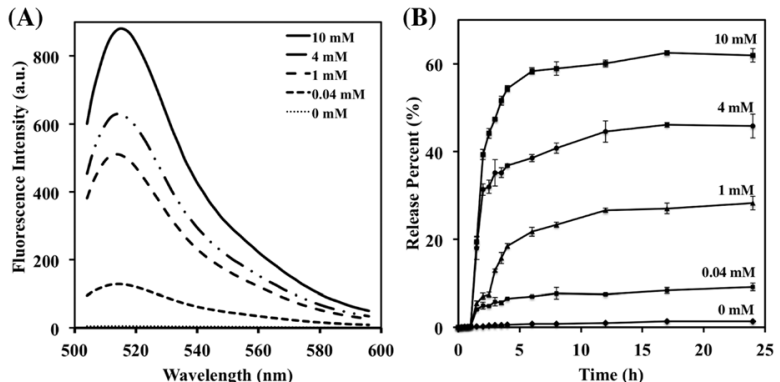

Fig. 2 (A) Emission spectra of FMSN-fConA in the presence of increasing concentrations of glutathione $\left(\lambda_{\mathrm{ex}}=490 \mathrm{~nm}, \lambda_{\mathrm{em}}=519 \mathrm{~nm}\right)$. (B) Release profiles of doxorubicin from FMSN-DOX-ConA nanoparticles in response to increasing concentrations of glutathione.

measured. As shown in Fig. 2A, no detectable fluorescence was recorded when the FMSN-fConA particles were dispersed in buffer in the absence of glutathione. In contrast, fluorescence appeared when glutathione was added to the nanoparticle suspension, where the intensity correlated with the glutathione concentrations. These results indicate that glutathione plays an effective role in cleaving the disulfide linker of the nanoparticles.

Absorption spectroscopy was used to monitor the release of doxorubicin from the Con A-gated glyconanoparticles in PBS ( $\mathrm{pH}$ 7.4) before and after exposure to the glutathione trigger. As shown in Fig. 2B, very low levels of doxorubicin were released within $24 \mathrm{~h}$ in the absence of glutathione, indicating that the lectin-gated nano-containers remained largely intact under these conditions. With increasing concentrations of glutathione from $0.04 \mathrm{mM}$ to $10 \mathrm{mM}$, on the other hand, the doxorubicin signal significantly increased, thus displaying lectin uncapping, pore opening, and drug release from the nanoparticles. The degree of doxorubicin release furthermore correlated with the glutathione concentrations. Thus, upon glutathione treatment, the disulfide linker was reduced and cleaved, resulting in removal of Con A from the surface of the FMSN-DOX-ConA particles, and release of doxorubicin. The process showed an initial burst phase over a few hours, followed by a prolonged period of slower release, likely due to diffusion from inner pores and potentially stronger adsorption to local surface sites. At a glutathione concentration of $10 \mathrm{mM}$, the release level within $7 \mathrm{~h}$ reached $61 \%$, similar to reported mesoporous particle systems. ${ }^{20}$ These results demonstrate that the lectin gating system showed efficient sealing properties before exposure to glutathione, and rapid opening and release of the nanoparticle cargo upon stimulation. Since cancer cells show high levels of glutathione compared to normal cells, the Con A-gated glyconanoparticles demonstrate high potential as drug carriers for delivery to cancer cells without premature release of drug into blood vessels and normal tissue. ${ }^{8 b, 21}$

The selective doxorubicin release efficiency of the Con A-gated glyconanoparticles towards human cells was next addressed. Cytotoxicity assays were thus performed with two different human lung cells lines: primary lung epithelial cells (PCC cells), and adenocarcinomic alveolar basal epithelial cells (A549 cells). The cells were treated with different concentrations of FMSN- and doxorubicin-loaded FMSN-DOX-ConA-particles, and the cell viability

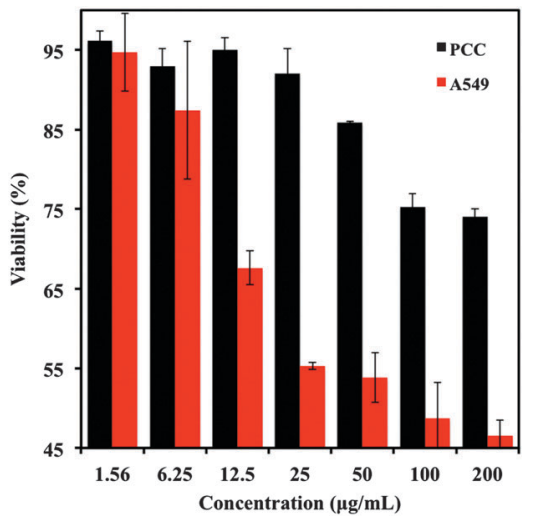

Fig. 3 Percent viability of A549 and PCC cells against FMSN-DOX-ConA particles.

over $24 \mathrm{~h}$ was recorded. As shown in Fig. 3, the cell selectivity proved very high, where the A549 cells were significantly more affected by the particles than the normal PCC cells. The IC50 of the FMSN-DOX-ConA particles against the A549 cells was approximately $25 \mu \mathrm{g} \mathrm{mL}{ }^{-1}$, while the viability of the normal PCC cells remained above $90 \%$ at this concentration. This demonstrated the effect of the high glutathione concentration in the A549 cancer cells $(\sim 12 \mathrm{mM}),{ }^{22}$ compared to the normal PCC cells. The concentration of glutathione in PCC cells is lower than $1 \mathrm{mM},{ }^{23}$ resulting in considerably lower degree of disulfide cleavage and doxorubicin release. For both cell lines, the unloaded FITC-doped mesoporous silica nanoparticles did not show any significant cytotoxicity, where the cell viability remained beyond $90 \%$ at nanoparticle concentrations from $1.6 \mu \mathrm{g} \mathrm{mL} \mathrm{m}^{-1}$ to $200 \mu \mathrm{g} \mathrm{mL}^{-1}$, demonstrating good biocompatibility of the FMSN particles (Fig. S4, ESI $\dagger$ ).

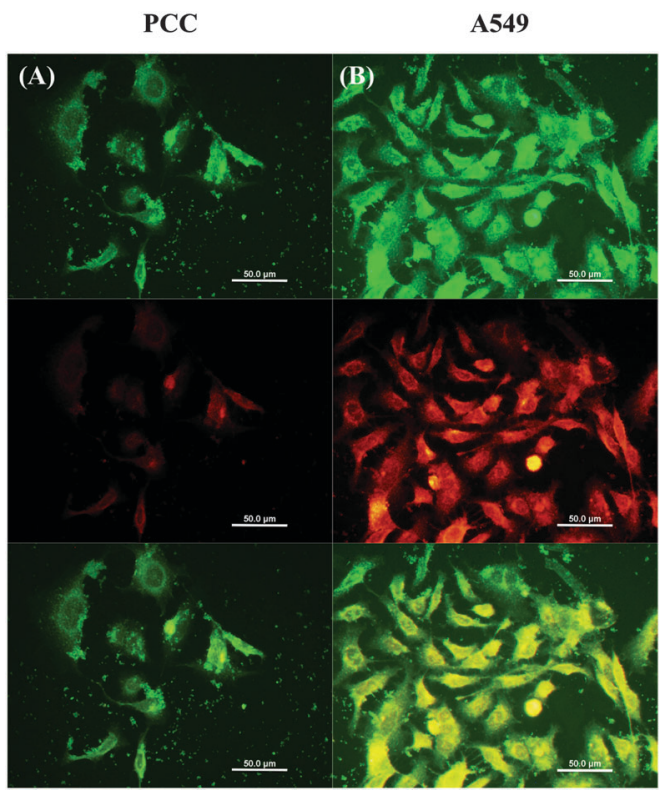

Fig. 4 Confocal microscope images of (A) PCC cells and (B) A549 cells incubated with FMSN-DOX-ConA particles; from top to bottom: emission measured at 500-530 nm (fluorescein), emission measured at 650-710 nm (doxorubicin), merged images showing both channels; scale bars $50 \mu \mathrm{m}$. 
The effects of the FMSN- and the FMSN-DOX-ConA particles on the cells were furthermore evaluated using confocal fluorescence microscopy. The cell lines were thus incubated with the different nanoparticles, and the fluorescence monitored in situ. The results were again clear, and the green fluorescence of the FITC-doped nanoparticles was visible in both the A549- and the PCC cells after incubation for $6 \mathrm{~h}$, indicating that the mesoporous silica nanoparticles can be uptaken by both types of human cells (Fig. 4, $c f$. Fig. S5, ESI $\dagger$ ). As can also be seen in Fig. 4, the A549 cells gave rise to strong red fluorescence upon incubation with the doxorubicin-loaded FMSN-DOX-ConA-particles. This was consistent with our expectation that extensive cleavage of the disulfide linker would occur with this cell line, leading to uncapping of the Con A gate, release of doxorubicin and appearance of red fluorescence. The results also support the effect of the higher concentration of glutathione in the A549 cells compared to the PCC cells, where doxorubicin can be released more efficiently from the Con A-gated nano-containers in the A549 cells. The weaker red fluorescence in the PCC cells suggests that the drug remained entrapped in the pores in the absence of high concentrations of glutathione. The developed delivery system thus showed a good blocking effect with minimal premature drug release in healthy cells.

In conclusion, this work describes the development of lectingated, FITC-doped mesoporous silica glyconanoparticles as delivery vehicles for controlled drug release. The particles were functionalized with disulfide-linked perfluorophenyl azide groups, and conjugated with D-mannose using photoinduced nitrene chemistry. Subsequent doxorubicin loading and Con A capping resulted in the final materials, which could be opened by redox stimulus. The lectin gatekeeper showed good blocking efficiency at low levels of glutathione in normal cells, while reduction of the disulfide linker in the presence of higher concentrations of glutathione in cancer cells efficiently uncapped the lectin and released the drug. These Con A-gated mesoporous silica glyconanoparticles provide an efficient platform for controlled drug delivery, in principle releasing the content at the site of disease. Further surface modification with targeting entities towards specific cells and tissues for site-specific controlled drug delivery is under consideration.

This work was in part supported by the National Institutes of Health (R01GM080295) and the Royal Institute of Technology. JZ thanks the China Scholarship Council for a special scholarship award.

\section{Notes and references}

1 (a) V. R. Devadasu, V. Bhardwaj and M. N. V. R. Kumar, Chem. Rev., 2013, 113, 1686-1735; (b) Z. Li, J. C. Barnes, A. Bosoy, J. F. Stoddart and J. I. Zink, Chem. Soc. Rev., 2012, 41, 2590-2605; (c) Q. He and J. Shi, Adv. Mater., 2014, 26, 391-411; (d) J. Yao, M. Yang and Y. Duan, Chem. Rev., 2014, 114, 6170-6178; (e) R. Mo, Q. Sun, J. Xue, N. Li, W. Li, C. Zhang and Q. Ping, Adv. Mater., 2012, 24, 3659-3665; $(f)$ G. Liu, L. Zhou, Y. Su and C.-M. Dong, Chem. Commun., 2014, 50, 12538-12541.

2 (a) J. E. Lee, N. Lee, T. Kim, J. Kim and T. Hyeon, Acc. Chem. Res., 2011, 44, 893-902; (b) F. M. Kievit and M. Zhang, Adv. Mater., 2011,
23, 217-247; (c) Y. Wang, M. S. Shim, N. S. Levinson, H.-W. Sung and Y. Xia, Adv. Funct. Mater., 2014, 24, 4206-4220; (d) S. Mura, J. Nicolas and P. Couvreur, Nat. Mater., 2013, 12, 991-1003.

3 (a) F. Tang, L. Li and D. Chen, Adv. Mater., 2012, 24, 1504-1534; (b) D. Tarn, C. E. Ashley, M. Xue, E. C. Carnes, J. I. Zink and C. J. Brinker, Acc. Chem. Res., 2013, 46, 792-801; (c) Q. He and J. Shi, J. Mater. Chem., 2011, 21, 5845-5855; (d) J. Lu, M. Liong, J. I. Zink and F. Tamanoi, Small, 2007, 3, 1341-1346; (e) B. G. Trewyn, S. Giri, I. I. Slowing and V. S. Y. Lin, Chem. Commun., 2007, 3236-3245; $(f)$ S.-H. Wu, Y. Hung and C.-Y. Mou, Chem. Commun., 2011, 47, 9972-9985.

4 (a) D. P. Ferris, Y.-L. Zhao, N. M. Khashab, H. A. Khatib, J. F. Stoddart and J. I. Zink, J. Am. Chem. Soc., 2009, 131, 1686-1688; (b) N. Fomina, C. McFearin, M. Sermsakdi, O. Edigin and A. Almutairi, J. Am. Chem. Soc., 2010, 132, 9540-9542; (c) J. Liu, W. Bu, L. Pan and J. Shi, Angew. Chem., Int. Ed., 2013, 52, 1-6.

5 (a) M. Xue, X. Zhong, Z. Shaposhnik, Y. Qu, F. Tamanoi, X. Duan and J. I. Zink, J. Am. Chem. Soc., 2011, 133, 8798-8801; (b) Y.-L. Zhao, Z. Li, S. Kabehie, Y. Y. Botros, J. F. Stoddart and J. I. Zink, J. Am. Chem. Soc., 2010, 132, 13016-13025.

6 (a) C. Liu, J. Guo, W. Yang, J. Hu, C. Wang and S. Fu, J. Mater. Chem., 2009, 19, 4764-4770; (b) B. Chang, X. Sha, J. Guo, Y. Jiao, C. Wang and W. Yang, J. Mater. Chem., 2011, 21, 9239-9247.

7 K. Patel, S. Angelos, W. R. Dichtel, A. Coskun, Y.-W. Yang, J. I. Zink and J. F. Stoddart, J. Am. Chem. Soc., 2008, 130, 2382-2383.

8 (a) J. Lai, B. P. Shah, E. Garfunkel and K.-B. Lee, ACS Nano, 2013, 7, 2741-2750; (b) H. Kim, S. Kim, C. Park, H. Lee, H. J. Park and C. Kim, Adv. Mater., 2010, 22, 4280-4283.

9 (a) M. J. Chen, C. S. Huang, C. S. He, W. P. Zhu, Y. F. Xu and Y. F. Lu, Chem. Commun., 2012, 48, 9522-9524; (b) D. Tang, B. Liu, R. Niessner, P. Li and D. Knopp, Anal. Chem., 2013, 85, 10589-10596; (c) S. Wu, X. Huang and X. Du, Angew. Chem., Int. Ed., 2013, 52, 5580-5584.

10 H. Lis and N. Sharon, Chem. Rev., 1998, 98, 637-674.

11 (a) M. A. Hayat, in Stains and Cytochemical Methods, Springer, New York, US, 1993, ch. 8, pp. 236-238; (b) K. D. Hardman and C. F. Ainsworth, Biochemistry, 1972, 11, 4910-4919.

12 (a) D. K. Mandal, N. Kishore and C. F. Brewer, Biochemistry, 1994, 33, 1149-1156; (b) O. Norberg, L. Deng, T. Aastrup, M. Yan and O. Ramström, Anal. Chem., 2011, 83, 1000-1007.

13 (a) J. Zhou, N. Butchosa, H. S. N. Jayawardena, Q. Zhou, M. Yan and O. Ramström, Bioconjugate Chem., 2014, 25, 640-643; (b) G. M. Edelman, B. A. Cunningham, G. N. Reeke, J. W. Becker, M. J. Waxdal and J. L. Wang, Proc. Natl. Acad. Sci. U. S. A., 1972, 69, 2580-2584.

14 P.-H. Liang, S.-K. Wang and C.-H. Wong, J. Am. Chem. Soc., 2007, 129, 11177-11184.

15 X. Chen, O. Ramström and M. Yan, Nano Res., 2014, 7, 1381-1403. 16 (a) G. K. Balendiran, R. Dabur and D. Fraser, Cell Biochem. Funct., 2004, 22, 343-352; (b) M. H. Lee, Z. Yang, C. W. Lim, Y. H. Lee, S. Dongbang, C. Kang and J. S. Kim, Chem. Rev., 2013, 113, 5071-5109.

17 (a) S. Huh, J. W. Wiench, J.-C. Yoo, M. Pruski and V. S. Y. Lin, Chem. Mater., 2003, 15, 4247-4256; (b) X. Wang, O. Ramström and M. Yan, Chem. Commun., 2011, 47, 4261-4263.

18 (a) L. Liu and M. Yan, Angew. Chem., Int. Ed., 2006, 45, 6207-6210; (b) O. Norberg, L. Deng, T. Aastrup, M. Yan and O. Ramström, Anal. Chem., 2011, 83, 1000-1007; (c) H. Wang, Y. Zhang, X. Yuan, Y. Chen and M. Yan, Bioconjugate Chem., 2011, 22, 26-32.

19 (a) X. Wang, E. Matei, L. Deng, O. Ramström, A. M. Gronenborn and M. Yan, Chem. Commun., 2011, 47, 8620-8622; (b) X. Wang, E. Matei, A. M. Gronenborn, O. Ramström and M. Yan, Anal. Chem., 2012, 84, 4248-4252.

20 J. Zhang, Z.-F. Yuan, Y. Wang, W.-H. Chen, G.-F. Luo, S.-X. Cheng, R.-X. Zhuo and X.-Z. Zhang, J. Am. Chem. Soc., 2013, 13, 5068-5073.

21 C. Park, H. Youn, H. Kim, T. Noh, Y. H. Kook, E. T. Oh, H. J. Park and C. Kim, J. Mater. Chem., 2009, 19, 2310-2315.

22 D. A. Keller and D. B. Menzel, Chem. -Biol. Interact., 1989, 70, 145-156.

23 K. Nagayoshi, T. Nemoto, S. Yokoyama, F. Yamashita and M. Hashida, Drug Metab. Pharmacokinet., 2004, 19, 407-412. 